Mohamed Salih, M. A. \& Markakis, John (eds). Ethnicity and the State in Eastern AfricaUppsala, Nordiska Afrikainstitutet, 1998, 208 p., index.

\title{
Alain Gascon
}

\section{OpenEdition}

\section{Journals}

Édition électronique

URL : http://journals.openedition.org/etudesafricaines/1554

DOI : 10.4000/etudesafricaines. 1554

ISSN : $1777-5353$

Éditeur

Éditions de l'EHESS

\section{Édition imprimée}

Date de publication : 1 janvier 2003

ISBN : 978-2-7132-1811-8

ISSN : 0008-0055

Référence électronique

Alain Gascon, « Mohamed Salih, M. A. \& Markakis, John (eds). - Ethnicity and the State in Eastern AfricaUppsala, Nordiska Afrikainstitutet, 1998, 208 p., index. », Cahiers d'études africaines [En ligne], 172 | 2003, mis en ligne le 02 mars 2007, consulté le 24 septembre 2020. URL : http://

journals.openedition.org/etudesafricaines/1554; DOI : https://doi.org/10.4000/etudesafricaines. 1554

Ce document a été généré automatiquement le 24 septembre 2020.

(c) Cahiers d'Études africaines 


\title{
Mohamed Salih, M. A. \& Markakis, John (eds). - Ethnicity and the State in Eastern AfricaUppsala, Nordiska Afrikainstitutet, 1998, 208 p., index.
}

\author{
Alain Gascon
}

1 Cet ouvrage collectif est publié par le Nordiska Afrikainstitutet (nai) d'Uppsala (Suède) qui s'est déjà signalé par son important travail d'édition. Il est dirigé par deux chercheurs chevronnés engagés dans des recherches remarquées: M. A. Mohamed Salih, anthropologue à l'Université de Khartoum, est responsable du programme de recherche "Human Life in African Arid Lands» au nai ; J. Markakis, professeur d'histoire de l'Afrique à l'Université de Crète (Rethymnon), est l'auteur d'ouvrages fondamentaux sur l'Éthiopie et la Corne de l'Afrique, plusieurs fois réédités ${ }^{1}$. Un premier reproche, et non le moindre, s'adresse plus à l'éditeur qu'aux auteurs et contributeurs : la carte de localisation qui suit la préface est indigne d'un livre destiné à un public « cultivé ». On conseillera au lecteur de se munir d'un atlas ou, à défaut, d'une carte routière d'Afrique de l'Est (Bartholomew ou Michelin). Les progrès des logiciels permettent, à peu de frais, d'exécuter des cartes lisibles mais la nouvelle n'a pas l'air d'avoir atteint les éditeurs, pas plus en Suède qu'ailleurs.

2 Le livre réunit les actes d'un atelier sur L'ethnicité et l'État organisé à Addis Abäba du 3 au 5 juin 1996 par le NAI et l'Organization for Social Science Research in Eastern and Southern Africa (OSSREA). Aidés par la Swedish International Development Agency (SIDA), les chercheurs éthiopiens y présentèrent, cinq ans après l'adoption du principe de l'ethnofédéralisme en Éthiopie, les travaux en cours parmi les groupes ethniques éthiopiens et les «nationalités ». D'autres chercheurs se joignirent à la réflexion au fur et à mesure de l'achèvement de la publication des actes. En effet, l'Éthiopie qui ignorait les appartenances «ethniques » ou les combattait décidait de se reconstruire sur une base fédérale «ethnique»: la Constitution de 1994 reconnaît à chaque nation, nationalité et peuple le droit à la sécession. Par la suite, les débats s'élargirent à 
l'ensemble de l'Afrique de l'Est dans un souci de comparatisme. Une première réflexion s'impose à la lecture de l'introduction de M. Salih \& J. Markakis : « Anathematized and outlawed by African nationalism early in the postcolonial period, ethnicity has now eclipsed nationalism in the continent" (p.7). L'Éthiopie n'a pas connu de période postcoloniale et a fait face pendant trente ans au soulèvement de l'Érythrée dont le coeur correspond aux hautes terres où est né l'antique royaume d'Aksum. En outre, de 1998 à 2000, les deux États ont soutenu une guerre où se sont affrontés deux nationalismes exacerbés. Or, les contributions ont été réunies à La Haye, en février 1998, et publiées avant le début du conflit. On peut augurer que les rapports entre ethnie et État ont été changés : évidemment, même dans les premiers mois de 1998, personne ne soupçonnait le prochain affrontement.

3 L'ouvrage se divise en deux parties d'importance à peu près égale, "Narratives of Ethnicity» et "Case Studies ». La moitié des contributions sont consacrées au cas éthiopien et elles sont situées au début des deux parties et introduisent la réflexion comparatiste. On doit noter que les auteurs des articles sur l'Éthiopie sont éthiopiens et färänj (étrangers), il en est de même pour les études consacrées au Soudan, au Kenya, à l'Ouganda, à la Tanzanie et au Tchad. Les éthiopisants ne seront pas surpris de retrouver les "nations, nationalités et peuples ", parmi les plus nombreux qui se sont disputés et se disputent toujours le pouvoir en Éthiopie : les Tegréens, les Oromo et les Amhara. Ils sont très heureux de lire, enfin, des travaux sur les Afar et les Guragé, des " petits peuples $»^{2}$ trop souvent ignorés. Ils profiteront des travaux sur les autres États où les conflits «ethniques» servent à expliquer la division des États (Soudan) ou au contraire leur unité par leur neutralisation réciproque (Kenya et Ouganda), les conflits frontaliers (Zaghawa) ou les vicissitudes des classements coloniaux en Tanzanie. Les éthiopisants et les lecteurs curieux seront surpris de ne lire aucune mention des termes dans les langues vernaculaires ${ }^{3}$. Ils trouveront dans les documents officiels en amharique: nation (behér), nationalités (behéräsäb) et peuple (hezb). D'autres mots existent comme agär (pays) souvent employé dans le sens de national. La Révolution a créé le néologisme behéräsäb. On ne sait pas comment les Éthiopiens traduisent « ethnie »: gosa (oromo) $/ g^{w a ̈ s} s$ (amharique) sont équivalents, selon les dictionnaires à tribu (tribe). Rien n'est mentionné, pas plus pour l'afar, pour le tigrigna que pour le guragé. Les études sont heureusement un peu plus précises au Kenya et chez les Zaghawa mais en Ouganda, Muhereza \& Otim utilisent people (p. 198). Certes, on ne peut prétendre à l'exhaustivité mais la transposition de notions d'une langue à une autre n'est pas une mince affaire notamment en Éthiopie, dépositaire d'une tradition de culture écrite sémitique.

4 Deux contributions, plus générales, se détachent dans l'ensemble de celles de M. Doornbos : «Linking the Future to the Past - Ethnicity and Pluralism» et d'E. Poluha : «Ethnicity and Democracy - A Viable Alliance?». Elles répondent aux cinq usages de l'ethnicité dans ses rapports avec l'État, tirés du cas éthiopien et répertoriés par les deux "éditeurs", dont deux d'entre eux ont une dimension spatiale: la régionalisation et le développement (p. 8). L'article de M. Doornbos examine l'importance de la mobilisation ethnique dans les luttes d'indépendance avec les dérives nationalistes et chauvines qui vont à l'encontre de la démocratisation espérée. E. Poluha s'attache à discuter le principe même de l'ethnofédéralisme comme principe unique et exclusif de recomposition politique et sociale en Éthiopie qui contraint les citoyens à n'avoir qu'une seule appartenance. Elle s'interroge sur le contenu ethnique des partis politiques de la coalition. L'ethnicisation de la république peut-elle aller 
jusqu'à son démembrement alors que l'éclatement territorial rencontre une opposition populaire menée par l'Église täwahedo (monophysite). Plus généralement, l'ethnisme ne serait-il pas le nouveau nom du nationalisme, un de ses avatars de plus. L'ethnisme ne serait-il pas le nationalisme de l'autre? E. Poluha n'avait pas la réponse à ces questions mais le conflit récent avec l'Érythrée a montré les limites du fédéralisme éthiopien comme l'écrit $\mathrm{M}$. Fontrier : " C'est bien une guérilla de culture marxiste qui, après avoir redécouvert les comportements politiques de ses anciens rois des rois, s'est fixé le devoir de mener l'Éthiopie sur la voie du libéralisme, de la modernité et, peut-être, de la démocratie $»^{4}$.

5 Les contributions suivantes se focalisent plus sur des études de cas même dans la généalogie des ethnismes, dans la formation des États. L'article de J. O'Brien remet en question bien des idées reçues sur le partage du Soudan selon l'opposition entre le Nord arabo-musulman et le Sud « africain » païen/chrétien. Il révèle que les migrations vers la Gazira et vers la capitale et la fuite devant la guerre ont conduit les Sudistes au Nord comme l'a montré la foule venue accueillir le Pape, à Khartoum, en $1993^{5}$. Des Sudistes qui parlent arabe et qui sont musulmans. M. Salih qui étudie la fondation du Front national islamique montre que l'alliance forcée, par le biais des confréries, entre arabisme et islam, rejette les musulmans non arabes vers l'opposition armée dont l'Armée de libération des peuples du Soudan (ALPS/SPLA ${ }^{6}$ ). Les articles de P. Baxter et de Adhana plaident pour les deux nationalités « opprimées " par la formation de la Grande Éthiopie sous le règne de Ménélik. De cette lutte pour la sauvegarde d'une culture commune est sortie pour le premier une nation oromo, l'Oromiyaa, et pour le second une nation, le Tegray, portée par le Front populaire de libération du Tegray (FPLT), au pouvoir depuis 1991. La contribution d'A.B.C. Ochollo-Ayayo met en avant une autre fonction de l'ethnicité : son rôle de régulation de la vie politique au Kenya, régulation ou instrumentalisation?

Dans la seconde partie, le cas éthiopien est repris dans l'esprit d'un livre qui fit beaucoup de bruit, qu'on oublia et qui connaît un regain d'intérêt depuis que des Éthiopiens l'ont traduit en amharique Greater Ethiopia de D. N. Levine ${ }^{7}$. Cette traduction est parue en réaction contre reconnaissance de l'ethnofédéralisme par la Constitution éthiopienne. P. Chanie, Ali Said, J. Markakis et Tegegne étudient respectivement les Oromo, les Afar, les Guragé et les Amhara. Ces auteurs mettent l'accent sur une des dimensions de la politisation de l'ethnicité : les luttes pour le pouvoir à l'intérieur des "ethnies" risquent de désagréger leur cohésion. Les Oromo, le peuple le plus nombreux d'Éthiopie (plus de 20 millions d'habitants), sont traversés de courants contraires depuis les grandes migrations des $\mathrm{XVII}^{\mathrm{e}}-\mathrm{XVIII}{ }^{\mathrm{e}}$ siècles. Les premières manifestations du réveil de l'identité des Oromo sont liées à une association volontaire de développement régional. Puis sous la Révolution centralisatrice, la conscience politique nationale des Oromo grandit en opposition à la politique de Mängestu. Avec l'institution du fédéralisme, les centres régionaux émergent de nouveau selon P. Chagnie et participent au pouvoir (OPDO) ou s'y opposent (FLO) ${ }^{8}$. Les clans afar poursuivent leurs conflits traditionnels, pour les pâturages, dans la lutte pour le pouvoir régional. La synthèse de J.Markakis est particulièrement pertinente: il annonce la montée des identités locales rivales. En 2001, le vote des Silté les a détachés de la zone Guragé. Or, cette consultation a obtenu l'accord du gouvernement qui possède ainsi un moyen de pression efficace sur les régions-États fédérales. La contribution de Tegegne insiste sur l'absence de conscience ethnique chez les Amhara 
au profit d'une conscience nationale car ils ont dominé l'Éthiopie jusqu'en 1991. On doit ajouter que le pouvoir amhara a noué des alliances politiques (et matrimoniales) avec les Tegréens, les Oromo, les Guragé... qui ont abouti à l'amharisation des élites locales par le biais de leur participation à l'administration, à l'armée et à l'Église. Ces études de cas permettent de penser l'ethnicisation à l'échelle locale et non plus seulement à l'échelon national.

7 L'étude de S. Harir montre comment les Zaghawa, partagés entre le Tchad et le Soudan, développent, à l'intérieur des États, des stratégies politiques de contrôle des pacages au mépris des règles traditionnelles d'accès aux ressources. On déplore que l'auteur ne mentionne aucun des travaux que J. et M.-J. Tubiana ont consacrés aux Zaghawa. La contribution de H.Jerman traite des transformations de la conscience ethnique provoquées par la colonisation chez les Bagamoyo de Tanzanie. L'ethnicité était un marqueur culturel que les colonisateurs et leur successeur ont utilisé pour distinguer des unités ethniques. Après l'indépendance, le gouvernement tanzanien a essayé de dépasser ces appartenances en promouvant une conscience nationale. Depuis son indépendance, l'Ouganda est miné par les conflits «ethniques» a-t-on souvent écrit. E. F. Baryamuga étudie comment, dans la suite de la période coloniale, les forces armées se sont peu à peu ethnicisées si bien que le président et le chef des armées appartiennent au même groupe ethnique ${ }^{9}$. L'article de F. E. Muherza et de P. E. Otim fait un bilan de la façon dont le régime de Yoweri Museveni cherche à dépasser les oppositions ethniques en instituant la régionalisation du pouvoir. Les développements récents des actions de l'Armée du Seigneur et les implications de l'armée ougandaise dans le conflit du Congo montrent que l'Ouganda se lance dans des conflits éloignés de toute référence ethnique. Museveni, un ancien maquisard, combat une guérilla, dans le premier cas, et dans l'aventure congolaise participe à la première guerre interafricaine.

Ce livre est intéressant au moins à deux titres : il fait le point sur les rapports entre la formation des États et le « réveil » des ethnies et c'est un témoignage sur la façon dont certains africanistes avaient pensé trouver dans l'« ethnie " l'une des clés de la compréhension des désordres politique du continent. Enfin, l'Éthiopie ethnofédérale rentrait dans le rang alors que l'Ouganda accomplissait le chemin inverse comme l'écrivent M. A. Mohamed Salih et J. Markakis dans leur introduction (p. 13). Or, le cas éthiopien n'est pas si simple et les contributeurs le montrent bien. Les articles d'E. Polhua, de P. Baxter, de P. Chanie et de J. Markakis sont, à cet égard, démonstratifs des difficultés à produire des bilans définitifs.

\section{NOTES}

1. Notamment : Ethiopia, Anatomy of a Traditional Polity, Oxford, Oxford University Press, 1974, et National and Class Conflict in the Horn of Africa, Cambridge, Cambridge University Press (« African Studies Series » 55), 1987.

2. Plus d'un million d'Afar et près de deux millions de Guragé ! 
3. Cf. mon article : « La corne de l'Afrique, terra incognita de l'ethnie? », in F. Dufaux \& P. Gervais-Lambony (dir.), Afrique noire-Europe de l'Est. Regards croisés, Paris, KarthalaGéotropiques, 1994 : 91-97.

4. «L'ethno-fédéralisme. Retour à un État ancien ", Les orientalistes sont des aventuriers. Guirlande offerte à Joseph Tubiana par ses élèves et ses amis, Textes réunis par A. Rouaud, Saint-Maur, Sépia, Bibliothèque Peiresc 12, 1999 : 215-222.

5. Témoignage de C. Delmet.

6. Sudan Peoples' Liberation Army de J. Garang.

7. Greater Ethiopia: The Evolution of a Multiethnic Society, Chicago and London, the University of Chicago Press, 2000 [1974].

8. L'Organisation populaire et démocratique des Oromo, basée au Wällägga chez les chrétiens, est au gouvernement tandis que le Front de libération des Oromo (chrétiens et musulmans) et les Fronts islamiques oromo le combattent.

9. Cf. La « Military Ethnocracy » d'Ali Mazrui, Soldiers and Kinsmen in Uganda, Beverly Hills, Sage, 1975. 